\title{
On replicator equations with nonlinear payoff functions defined by the Ricker models
}

\section{Sur les équations de réplicateur avec fonctions à gain non-linéaire définies par les modèles de Ricker}

\author{
Mansoor Saburov \\ College of Engineering and Technology, American University of the Middle East, Kuwait \\ msaburov@gmail.com, mansur.saburov@aum.edu.kw
}

\begin{abstract}
An evolutionary game is usually identified by a smooth (possibly nonlinear) payoff function. In this paper, we propose a model of evolutionary game in which the nonlinear payoff functions are defined by the Ricker models. Namely, in the proposed model, the biological fitness of the pure strategy will increase according to the Ricker model. Motivated by some models of economics of transportation and communication networks, in order to observe the evolutionary bifurcation diagram, we also control the nonlinear payoff functions in two different regimes: positive and negative. One of the interesting feature of the model is that if we switch the controlling parameter from positive to negative regime then the set of local evolutionarily stable strategies (ESSs) changes from one set to another one. We also study the dynamics and stability analysis of the discrete-time replicator equation governed by the proposed nonlinear payoff function. In the long-run time, the following scenario can be observed: (i) in the positive regime, the active dominating pure strategies will outcompete other strategies and only they will survive forever; (ii) in the negative regime, all active pure strategies will coexist together and they will survive forever.

Mathematics Subject Classification (2010). 91A22, 37N25, 92D25.

KEYWORDS. Replicator Equation, Evolutionarily stable strategy, Nash equilibrium, Ricker model
\end{abstract}

\section{Introduction}

Evolutionary game theory is the study of frequency-dependent natural selection in which the fitness of individuals is not constant, but depends on frequencies of the different phenotypes in population. It encompasses into itself competition (the game), natural selection (replicator dynamics), and heredity which contributes to the understanding of group selection, sexual selection, altruism, parental care, co-evolution, and ecological dynamics. This theory was introduced by John Maynard Smith and George R. Price in a paper [18] published in Nature in 1973 and further developed by John Maynard Smith in his seminal book [17] "Evolution and the Theory of Games" published in 1982 (see also [16]). Since then, there has been a veritable explosion of interest by economists and social scientists in evolutionary game theory $[4,5,7,8,11-13,22,31,33,40]$.

Classical non-cooperative game theory, which was invented by John von Neumann and Oskar Morgenstern (see [39]) in 1944, typically analyzes an interaction between two players and studies their behavior in strategic and economic decisions. The main problem is how the players can maximize their payoffs in a game given that the players are aware of the structure of the game and consciously try to predict the moves of their opponents. This depends on the cognitive abilities of the players in which the concept of rationality plays an important role. The Nash equilibrium, which was invented by John Nash (see $[20,21])$ in 1950, is the solution concept in game theory. It is a strategy profile in which no player can do better by unilaterally changing their strategy to another strategy. In other words, each strategy in the Nash equilibrium is the best response to all other strategies in that equilibrium. 
Evolutionary game theory differs from classical game theory in focusing more on the dynamics of strategy change. It does not rely on rationality. It is presumed that a population of players are randomly interacted and the strategy of a player is fixed which is biologically encoded and heritable that controls its action. However, they have no control over their strategy and need not to be aware of the game. In other words, the players do not choose their strategy and cannot change it: they are born with a strategy and their offspring inherit that same strategy. It is also imagined that alternative strategies of the game occasionally occur via a process like mutation. Evolutionary game theory interprets payoff as biological fitness and success as reproductive success. Hence, the payoff represents reproductive success and the success of a strategy is determined by how good the strategy is in the presence of competing strategies and of the frequency with which those strategies are used. Strategies that do well reproduce faster and strategies that do poorly are outcompeted. This is straightforward natural selection.

An evolutionarily stable strategy (ESS) is akin to the Nash equilibrium in classical game theory. An ESS is a strategy of game dynamics for which another mutant strategy cannot successfully enter the population to disturb the existing dynamic. If it is adopted by a population in a given environment then it is unbeatable which means that it cannot be invaded by any alternative (mutant) strategy that are initially rare. The ESS is an equilibrium refinement of the Nash equilibrium that is "evolutionarily" stable: once it is fixed in a population, natural selection alone is sufficient to prevent alternative (mutant) strategies from invading successfully. The ESS must be resistant to these alternatives. Therefore, the ESS must be both effective against any alternative (mutant) strategy when it is initially rare and successful when it is eventually abundant. This means that the ESS must be successful when it contends with others exactly like itself.

The primary way to study the evolutionary dynamics in games is through replicator equations. They are used to describes the evolutionary dynamics of an entity called replicator which has means of making more or less accurate copies of itself. The replicator can be a gene in population genetics, a molecule in prebiotic evolution, an organism in population ecology, a strategy in evolutionary game. The replicator equation, which was first introduced into models of animal behavior of a single species by Peter D. Taylor and Leo B. Jonker (see [36]) in 1978 (see also [35]) and named by Peter Schuster and Karl Sigmund (see [32]) in 1983, is the cornerstone of evolutionary game dynamics. The replicator equation shows the growth rate of the proportion of players using a certain strategy and that rate is equal to the difference between the average payoff of that strategy and the average payoff of the population as a whole. The general idea is that replicators whose fitness is larger (smaller) than the average fitness of population will increase (decrease) in numbers. The static approach of evolutionary game theory has been complemented by a dynamic stability analysis of rest (stationary) solutions of the replicator equations. The replicator equation satisfies the folk theorem of evolutionary game theory which characterizes the stability of its rest (stationary) solutions. The stable stationary solutions are often given by the set of evolutionarily stable strategies (ESSs) of the population.

In this paper, we study the dynamics and stability analysis of the discrete-time replicator equation governed by a model of evolutionary game in which the nonlinear payoff functions are defined by the Ricker models. Namely, in the proposed model, the biological fitness of the pure strategy will increase according to the Ricker model. By motivated some models of the economics of transportation, in order to observe the evolutionary bifurcation diagram, the nonlinear payoff functions are controlled in two different regimes: positive and negative. We describe all fixed (rest) points, Nash equilibria, and ESSs of the proposed discrete-time replicator equation in both regimes. One of the interesting feature of the 
model is that if we switch the controlling parameter from positive to negative regime then the set of ESSs changes from one set to another one. In the long-run time, the following scenario can be observed: (i) in the positive regime, the active dominating pure strategies will outcompete other strategies and only they will survive forever; (ii) in the negative regime, all active pure strategies will coexist together and they will survive forever.

The paper is organized as follows: in the next section, we provide all necessary notions and notation from evolutionary game theory which will be used throughout this paper. In Section 3, we first propose a model of evolutionary game in which the nonlinear payoff functions are defined by the Ricker models and we then provide a discrete-time replicator equation defined by the proposed nonlinear payoff functions. We also describe the sets of all fixed (rest) points and Nash equilibria of the proposed model. In Sections 4 and 5, we first describe the set of all local ESSs of the nonlinear payoff mapping and we then study the dynamics and stability analysis of the discrete-time replicator equation in positive and negative regimes, respectively. Finally, we finish this paper with some concluding remarks in Section 6.

\section{Preliminaries}

In this paper, we consider a symmetric two-player normal form game that is a game of a single population (no difference of being player-1 or player-2) in which all players share the same strategy set and payoff function. Namely, we consider a unit mass of players each of whom chooses a pure strategy from the set $\mathbf{I}_{m}=\{1,2, \cdots, m\}$. A mixed strategy is a probability vector $\mathbf{x}=\left(x_{1}, \cdots, x_{m}\right)$, i.e., $x_{1}+\cdots+x_{m}=1$ and $x_{i} \geq 0$ for all $i \in \mathbf{I}_{m}$ where $x_{i}$ is the probability that the player will choose the pure strategy $i$. A pure strategy $i$ can be also seen as a mixed strategy $\mathbf{e}_{i}=(0, \cdots, 0,1,0, \cdots, 0)$ with 1 at the $i$ th place meaning that the player uses the strategy $i$ with probability 1 . The set of mixed strategy of player is the simplex $\mathbb{S}^{m-1}=\left\{\mathbf{x} \in \mathbb{R}_{+}^{m}: \sum_{i=1}^{m} x_{i}=1\right\}$. Sometimes, the simplex $\mathbb{S}^{m-1}$ can be also considered as the set of population states which describe densities of the population playing pure strategies. Namely, a probability vector $\mathbf{x}=\left(x_{1}, x_{2}, \cdots, x_{m}\right)$ is a population state where $x_{i}$ is the frequency of the strategy $i$.

A continuous (possibly nonlinear) function $f_{i}(\mathbf{x})$ is a payoff to strategy $i \in \mathbf{I}_{m}$ in a population state $\mathbf{x} \in \mathbb{S}^{m-1}$. A continuous mapping $F(\mathbf{x}):=\left(f_{1}(\mathbf{x}), \cdots, f_{m}(\mathbf{x})\right)$ is a payoff of the game when the population state is $\mathbf{x} \in \mathbb{S}^{m-1}$. A payoff to the strategy $\mathbf{y} \in \mathbb{S}^{m-1}$ when the population state is $\mathbf{x} \in \mathbb{S}^{m-1}$ is a bivariate continuous function $\mathcal{E}_{F}(\mathbf{y}, \mathbf{x}):=\sum_{i=1}^{m} y_{i} f_{i}(\mathbf{x})$ which is linear in the first argument. It is easy to see that $\mathcal{E}_{F}\left(\mathbf{e}_{i}, \mathbf{x}\right)=f_{i}(\mathbf{x})$ for any $i \in \mathbf{I}_{m}$. A average fitness of the population when it is a state $\mathbf{x} \in \mathbb{S}^{m-1}$ is $\mathcal{E}_{F}(\mathbf{x}, \mathbf{x})=\sum_{i=1}^{m} x_{i} f_{i}(\mathbf{x})$.

Sometimes, we say that the strategy $\mathbf{y} \in \mathbb{S}^{m-1}$ is better reply to the strategy $\mathbf{x} \in \mathbb{S}^{m-1}$ than the strategy $\mathbf{z} \in \mathbb{S}^{m-1}$ if one has $\mathcal{E}_{F}(\mathbf{y}, \mathbf{x})>\mathcal{E}_{F}(\mathbf{z}, \mathbf{x})$. The strategy $\mathbf{y} \in \mathbb{S}^{m-1}$ is called a best reply to the strategy $\mathbf{x} \in \mathbb{S}^{m-1}$ if one has $\mathcal{E}_{F}(\mathbf{y}, \mathbf{x}) \geq \mathcal{E}_{F}(\mathbf{z}, \mathbf{x})$ for any $\mathbf{z} \in \mathbb{S}^{m-1}$. The set of all best replies to $\mathbf{x} \in \mathbb{S}^{m-1}$ is denoted by

$$
\operatorname{BR}(\mathbf{x}):=\left\{\mathbf{y} \in \mathbb{S}^{m-1}: \mathcal{E}_{F}(\mathbf{y}, \mathbf{x})=\max _{\mathbf{z} \in \mathbb{S}^{m-1}} \mathcal{E}_{F}(\mathbf{z}, \mathbf{x})\right\} .
$$

Definition 2.1 (Nash Equilibrium). A strategy $\mathbf{x} \in \mathbb{S}^{m-1}$ is called a Nash equilibrium if it is a best reply to itself, i.e., one has $\mathcal{E}_{F}(\mathbf{x}, \mathbf{x}) \geq \mathcal{E}_{F}(\mathbf{y}, \mathbf{x})$ for any $\mathbf{y} \in \mathbb{S}^{m-1}$. A strategy $\mathbf{x}$ is called a strict Nash equilibrium if it is the unique best reply to itself, i.e., one has $\mathcal{E}_{F}(\mathbf{x}, \mathbf{x})>\mathcal{E}_{F}(\mathbf{y}, \mathbf{x})$ for any $\mathbf{y} \in \mathbb{S}^{m-1}$ with $\mathbf{y} \neq \mathbf{x}$.

It was pointed out by Heinz-Joachim Pohley and Bernhard Thomas in the paper [25] that the original 
definition of an evolutionarily stable strategy (ESS) is inappropriate for the games with nonlinear payoff functions. The main reason for this was the global character of the original conditions that define an ESS. These conditions always cover complete spaces of strategies which do not allow to have more than one ESS in the interior of the whole space. In the series of papers [25, 37, 38] (for further developments also see [2, 34]), in order to avoid such constraints, the concept of a local ESS was introduced for the games with nonlinear payoff functions for which the conditions that define an ESS must hold only locally, i.e. within a small neighborhood of an ESS. It turns out that for linear models the local ESS is equivalent to the original global ESS (see [25]).

Definition 2.2 (Local ESS). A strategy $\mathbf{x} \in \mathbb{S}^{m-1}$ is called a local evolutionarily stable strategy (a local ESS) if the following conditions hold true:

(i) $\mathbf{x}$ is a Nash equilibrium, i.e., $\mathcal{E}_{F}(\mathbf{x}, \mathbf{x}) \geq \mathcal{E}_{F}(\mathbf{y}, \mathbf{x})$ for any $\mathbf{y} \in \mathbb{S}^{m-1}$;

(ii) There is a small neighborhood $U(\mathbf{x}) \subset \mathbb{S}^{m-1}$ of $\mathbf{x}$ such that $\mathcal{E}_{F}(\mathbf{x}, \mathbf{y})>\mathcal{E}_{F}(\mathbf{y}, \mathbf{y})$ for all $\mathbf{y} \in U(\mathbf{x}) \backslash\{\mathbf{x}\}$.

The replicator equation which was originally developed for symmetric games with finitely many strategies (see [35, 36]) is the most important evolutionary game dynamics. It satisfies the folk theorem of evolutionary game theory which characterizes the stability of ESSs of the population.

In this paper, we consider a discrete-time replicator equation $\mathcal{R}_{F}: \mathbb{S}^{m-1} \rightarrow \mathbb{S}^{m-1}$

$$
\left(\mathcal{R}_{F}(\mathbf{x})\right)_{k}=x_{k}\left(1+f_{k}(\mathbf{x})-\mathcal{E}_{F}(\mathbf{x}, \mathbf{x})\right), \quad \forall \mathbf{x} \in \mathbb{S}^{m-1} \text { and } k \in \mathbf{I}_{m} .
$$

In the replicator equation, the growth rate of the player using a certain strategy is equal to the difference between the average payoff of that strategy and the average payoff of the population as a whole. The key idea is that replicators whose fitness is larger (smaller) than the average fitness of population will increase (decrease) in numbers. In order to study the stability of rest solutions (fixed points) of the replicator equation, we employ the Lyapunov function.

Definition 2.3 (Lyapunov Function). A continuous function $\varphi: \mathbb{S}^{m-1} \rightarrow \mathbb{R}$ is called a Lyapunov function if the number sequence $\left\{\varphi(\mathbf{x}), \varphi(\mathcal{R}(\mathbf{x})), \cdots, \varphi\left(\mathcal{R}^{(n)}(\mathbf{x})\right), \cdots\right\}$ is a bounded monotone sequence for any initial point $\mathbf{x} \in \mathbb{S}^{m-1}$.

We use the following notions and notations through out this paper.

Some Notations: Let $\mathbf{x}=\left(x_{1}, \cdots, x_{m}\right) \in \mathbb{R}^{m}$ and $\|\mathbf{x}\|_{1}:=\sum_{k=1}^{m}\left|x_{k}\right|$. We say that $\mathbf{x} \geq 0$ (resp. $\mathbf{x}>0$ ) if $x_{k} \geq 0$ (resp. $x_{k}>0$ ) for all $k \in \mathbf{I}_{m}$. We set $(\mathbf{x}, \mathbf{y}):=\sum_{i=1}^{m} x_{i} y_{i}$ for any $\mathbf{x}, \mathbf{y} \in \mathbb{R}^{m}$. Let $\mathbb{S}^{m-1}=\left\{\mathbf{x} \in \mathbb{R}^{m}\right.$ : $\left.\|\mathbf{x}\|_{1}=1, \mathbf{x} \geq 0\right\}$ be the standard simplex. We let $\operatorname{supp}(\mathbf{x}):=\left\{i \in \mathbf{I}_{m}: x_{i} \neq 0\right\}$ and null $(\mathbf{x}):=\left\{i \in \mathbf{I}_{m}: x_{i}=0\right\}$ for $\mathbf{x} \in \mathbb{S}^{m-1}$. The vertex $\mathbf{e}_{i}:=(0, \cdots, 0,1,0, \cdots, 0)$ with 1 at the $i$ th place is the pure strategy $i \in \mathbf{I}_{m}$. Let $\mathbb{S}^{|\alpha|-1}:=\operatorname{conv}\left\{\mathbf{e}_{i}\right\}_{i \in \alpha}$ for $\alpha \subset \mathbf{I}_{m}$ where $\operatorname{conv}(\mathbf{A})$ is the convex hull of $\mathbf{A}$. Let int $\mathbb{S}^{|\alpha|-1}:=\left\{\mathbf{x} \in \mathbb{S}^{|\alpha|-1}\right.$ : $\operatorname{supp}(\mathbf{x})=\alpha\}$ and $\partial \mathbb{S}^{|\alpha|-1}:=\mathbb{S}^{|\alpha|-1} \backslash \operatorname{int} \mathbb{S}^{|\alpha|-1}$ be, respectively, an interior and boundary of the face $\mathbb{S}^{|\alpha|-1}$. The center $\mathbf{e}_{\alpha}:=\frac{1}{|\alpha|} \sum_{i \in \alpha} \mathbf{e}_{i}$ of the face $\mathbb{S}^{|\alpha|-1}$ is the equally distributed population state of the active pure strategies $i \in \alpha$. We define a function $\mathcal{M}_{\alpha, k}(\mathbf{x}):=\max _{i \in \alpha}\left\{x_{i}\right\}-x_{k}$ for $k \in \alpha \subset \mathbf{I}_{m}$. Particularly, when $\alpha=\mathbf{I}_{m}$, we write $\mathcal{M}_{k}(\mathbf{x}):=\max _{i \in \mathbf{I}_{m}}\left\{x_{i}\right\}-x_{k}$ for $k \in \mathbf{I}_{m}$. We define the sets $\operatorname{MaxInd}_{\alpha}(\mathbf{x}):=\left\{k \in \alpha: x_{k}=\max _{i \in \alpha}\left\{x_{i}\right\}\right\}$ 
and $\operatorname{MinInd}_{\alpha}(\mathbf{x}):=\left\{k \in \alpha: x_{k}=\min _{i \in \alpha}\left\{x_{i}\right\}\right\}$ for $\alpha \subset \mathbf{I}_{m}$. Particularly, we write MaxInd( $\left.\mathbf{x}\right)$ and Minlnd( $(\mathbf{x})$ for the set $\alpha=\mathbf{I}_{m}$. An orbit (trajectory) of an initial point $\mathbf{x} \in \mathbb{S}^{m-1}$ is defined as $\left\{\mathbf{x}, \mathcal{R}(\mathbf{x}), \cdots, \mathcal{R}^{(n)}(\mathbf{x}), \cdots\right\}$. An omega limiting set $\omega(\mathbf{x})$ of the orbit is defined as $\omega(\mathbf{x}):=\bigcap_{n \in \mathbb{N}} \overline{\bigcup_{k \geq n}\left\{\mathcal{R}^{(k)}(\mathbf{x})\right\}}$. A fixed (rest) point set of the replicator operator is $\mathbf{F i x}(\mathcal{R})=\left\{\mathbf{x} \in \mathbb{S}^{m-1}: \mathcal{R}(\mathbf{x})=\mathbf{x}\right\}$. We denote by $\mathbf{N E}(F), \mathbf{S N E}(F)$ and $\mathbf{E S S}(F)$ the sets of, respectively, all Nash equilibria, all strict Nash equilibria, and all local ESSs of the payoff mapping $F$.

\section{Replicator equations defined by the Ricker models}

It is worth mentioning that in some models of economics of transportation (see [3, 28-31]) and communication networks (see [1, 6, 14, 15, 23, 24]) it is plausible to consider payoff functions which take on only negative values. For the sake of discussion, we provide allocation problems of flows in a communication network which serve as motivational examples in our study. We adapt notions and notations from the papers [1, 23] (for a general study see [6, 14, 15, 24]).

We consider a network with $m$ parallel links (edges) $\mathbf{I}_{m}=\{1,2, \cdots, m\}$ that connect a single sourcedestination pair. We are interested in the problem of routing one unit of flow across $m$ alternative parallel links (routes). We shall use the terms route and link interchangeable. Let $x_{k}$ denote the total flow on route $k$ and $\mathbf{x}=\left(x_{1}, x_{2}, \cdots, x_{m}\right)$ denote a vector of rout flows. Obviously, we have $x_{1}+x_{2}+\cdots+x_{m} \leq 1$ and $x_{k} \geq 0$ for all $k \in \mathbf{I}_{m}$. In the routing problem, a journey time is the main component of a travel cost. The route journey times increase when the load of flow becomes heavier. Each link $k$ in the network has a flowdependent latency function $l_{k}\left(x_{k}\right)$ which measures the journey time as a function of the total flow $x_{k}$ on link $k$. We assume that for each $k \in \mathbf{I}_{m}$ the latency function $l_{k}:[0, \infty) \rightarrow[0, \infty)$ is a convex, strictly increasing, continuously differentiable function such that $l_{k}(0)=0$. Let $L(\mathbf{x})=\left(l_{1}\left(x_{1}\right), l_{2}\left(x_{2}\right), \cdots, l_{m}\left(x_{m}\right)\right)$ denote a latency mapping at a flow distribution $\mathbf{x}$. Assume that each link $k$ is owned by a service provider who sets a per unit bandwidth (flow) price of $p_{k}$. Let $\mathbf{p}=\left(p_{1}, p_{2}, \cdots, p_{m}\right)$ denote a vector of rout prices. In order to choose the amount of flow and the routing pattern optimally, we define an effective cost of using route $k$ to be the sum of the latency $l_{k}\left(x_{k}\right)$ and the route price $p_{k}$ when the total flow on route $k$ is $x_{k}$, i.e., $l_{k}\left(x_{k}\right)+p_{k}$. Let $L(\mathbf{x})+\mathbf{p}$ denote an effective cost vector at a flow distribution $\mathbf{x}$. We also assume that a common reservation utility is $R$ and it is decided not to send a flow when the effective cost of route exceeds the reservation utility. Let $\mathbf{R}=(R, R, \cdots, R)$ denote a reservation utility vector. A central question is whether prices chosen to maximize service provider revenues will also play a useful role in an optimal allocation of network resources across users. Namely, the profit-maximizing service providers own the routes in a communication network and set prices to maximize their profits, while selfish-users choose the amount of flow to send and the routing pattern optimally. In order to characterize an optimal flow distribution in a communication network, Wardrop's principle (equilibrium) is adopted: the effective costs on all utilized links are equal and minimal. It is worth mentioning (see [1, 23]) that for any $\mathbf{p} \in \mathbb{R}_{+}^{m}$ with $\mathbf{p} \leq \mathbf{R}$ there always exists a unique Wardrop equilibrium $\mathbf{x}_{\mathbf{p}}=\left(x_{1}^{(\mathbf{p})}, x_{2}^{(\mathbf{p})}, \cdots, x_{m}^{(\mathbf{p})}\right) \in \mathbb{R}_{+}^{m}$ with either one of the following properties:

$$
\begin{array}{ll}
l_{i}\left(x_{i}^{(\mathbf{p})}\right)+p_{i}=\min _{k \in \mathbf{I}_{m}}\left\{l_{k}\left(x_{k}^{(\mathbf{p})}\right)+p_{k}\right\}<R, & \forall i \in \operatorname{supp}\left(\mathbf{x}_{\mathbf{p}}\right), \quad\left\|\mathbf{x}_{\mathbf{p}}\right\|_{1}=1, \\
l_{i}\left(x_{i}^{(\mathbf{p})}\right)+p_{i}=\min _{k \in \mathbf{I}_{m}}\left\{l_{k}\left(x_{k}^{(\mathbf{p})}\right)+p_{k}\right\}=R, & \forall i \in \operatorname{supp}\left(\mathbf{x}_{\mathbf{p}}\right), \quad\left\|\mathbf{x}_{\mathbf{p}}\right\|_{1} \leq 1 .
\end{array}
$$


It is easy to prove (see [1]) that the unique Wardrop equilibrium $\mathbf{x}_{\mathbf{p}} \in \mathbb{R}_{+}^{m}$ is a solution to the following optimization problem:

$$
\mathbf{x}_{\mathbf{p}} \in \underset{\substack{\mathbf{y} \geq 0 \\\|\mathbf{y}\|_{1} \leq 1}}{\operatorname{Argmax}}\left\{\sum_{k=1}^{m}\left(R-l_{k}\left(x_{k}^{(\mathbf{p})}\right)-p_{k}\right) y_{k}\right\}
$$

We now show that for any $\mathbf{p}=(p, p, \cdots, p)$ with $p<R$ the unique Wardrop equilibrium $\mathbf{x}_{\mathbf{p}}$ with $\left\|\mathbf{x}_{\mathbf{p}}\right\|_{1}=1$ is a Nash equilibrium of the payoff mapping

$$
-L(\mathbf{x})=\left(-l_{1}\left(x_{1}\right),-l_{2}\left(x_{2}\right), \cdots,-l_{m}\left(x_{m}\right)\right) .
$$

Indeed, it follows from the optimization problem that

$$
\sum_{k=1}^{m}\left(R-l_{k}\left(x_{k}^{(\mathbf{p})}\right)-p\right) x_{k}^{(\mathbf{p})} \geq \sum_{k=1}^{m}\left(R-l_{k}\left(x_{k}^{(\mathbf{p})}\right)-p\right) y_{k}, \quad \forall \mathbf{y} \geq 0, \quad\|\mathbf{y}\|_{1} \leq 1 .
$$

Since $\left\|\mathbf{x}_{\mathbf{p}}\right\|_{1}=1$, we obtain that

$$
R-p+\mathcal{E}_{-L}\left(\mathbf{x}_{\mathbf{p}}, \mathbf{x}_{\mathbf{p}}\right) \geq(R-p) \sum_{k=1}^{m} y_{k}+\mathcal{E}_{-L}\left(\mathbf{y}, \mathbf{x}_{\mathbf{p}}\right), \quad \forall \mathbf{y} \geq 0,\|\mathbf{y}\|_{1} \leq 1 .
$$

Particularly, we have that $\mathcal{E}_{-L}\left(\mathbf{x}_{\mathbf{p}}, \mathbf{x}_{\mathbf{p}}\right) \geq \mathcal{E}_{-L}\left(\mathbf{y}, \mathbf{x}_{\mathbf{p}}\right)$ for all $\|\mathbf{y}\|_{1}=1$ with $\mathbf{y} \geq 0$. This means that for any $\mathbf{p}=(p, p, \cdots, p)$ with $p<R$ the unique Wardrop equilibrium $\mathbf{x}_{\mathbf{p}}$ with $\left\|\mathbf{x}_{\mathbf{p}}\right\|_{1}=1$ is a Nash equilibrium.

It is worth mentioning that a Wardrop equilibrium is well-known and frequently applied in transportation and communication networks (see [1, 3, 6, 14, 15, 23]). It is intuitively very appealing and has had successful applications in a wide variety of social sciences. For a thorough review of the Wardrop equilibrium, one can refer to the following monograph [24].

We now provide some sufficient condition which assures an existence of Wardrop equilibrium inside the simplex $\mathbb{S}^{m-1}=\left\{\mathbf{x} \in \mathbb{R}^{m}:\|\mathbf{x}\|_{1}=1, \mathbf{x} \geq 0\right\}$.

Proposition 3.1. Let $\left(p_{1}, p_{2}, \cdots, p_{m}\right)=\mathbf{p}<\mathbf{R}=(R, R, \cdots, R)$ and $l_{k}^{-1}$ be the inverse function of $l_{k}$. Then the following statements are true:

- If $\sum_{k=1}^{m} l_{k}^{-1}\left(R-p_{k}\right)<1$ then $\left\|\mathbf{x}_{\mathbf{p}}\right\|_{1}<1$;

- If $\sum_{k=1}^{m} l_{k}^{-1}\left(R-p_{k}\right) \geq 1$ then $\left\|\mathbf{x}_{\mathbf{p}}\right\|_{1}=1$.

Proof. Indeed, if $\sum_{k=1}^{m} l_{k}^{-1}\left(R-p_{k}\right) \leq 1$ then the unique Wardrop equilibrium is

$$
\mathbf{x}_{\mathbf{p}}=\left(l_{1}^{-1}\left(R-p_{1}\right), l_{2}^{-1}\left(R-p_{2}\right), \cdots, l_{m}^{-1}\left(R-p_{m}\right)\right)
$$

with $l_{i}\left(x_{i}^{(\mathbf{p})}\right)+p_{i}=\min _{k \in \mathbf{I}_{m}}\left\{l_{k}\left(x_{k}^{(\mathbf{p})}\right)+p_{k}\right\}=R, \forall i \in \mathbf{I}_{m}$ and $\left\|\mathbf{x}_{\mathbf{p}}\right\|_{1}=\sum_{k=1}^{m} l_{k}^{-1}\left(R-p_{k}\right)$.

Let $\sum_{k=1}^{m} l_{k}^{-1}\left(R-p_{k}\right)>1$. If it is assumed that

$$
l_{i}\left(x_{i}^{(\mathbf{p})}\right)+p_{i}=\min _{k \in \mathbf{I}_{m}}\left\{l_{k}\left(x_{k}^{(\mathbf{p})}\right)+p_{k}\right\}=R, \quad \forall i \in \operatorname{supp}\left(\mathbf{x}_{\mathbf{p}}\right)
$$


we then obtain that $\operatorname{supp}\left(\mathbf{x}_{\mathbf{p}}\right)=\mathbf{I}_{m}$ because of $l_{k}(0)=0$ and $p_{k}<R$ for all $k \in \mathbf{I}_{m}$. Therefore, we have $x_{k}^{(\mathbf{p})}=l_{k}^{-1}\left(R-p_{k}\right), \forall k \in \mathbf{I}_{m}$ and $\left\|\mathbf{x}_{\mathbf{p}}\right\|_{1}=\sum_{k=1}^{m} l_{k}^{-1}\left(R-p_{k}\right)>1$. This contradiction tells that we must have

$$
l_{i}\left(x_{i}^{(\mathbf{p})}\right)+p_{i}=\min _{k \in \mathbf{I}_{m}}\left\{l_{k}\left(x_{k}^{(\mathbf{p})}\right)+p_{k}\right\}<R, \quad \forall i \in \operatorname{supp}\left(\mathbf{x}_{\mathbf{p}}\right)
$$

which yields $\left\|\mathbf{x}_{\mathbf{p}}\right\|_{1}=1$. Consequently, if $\sum_{k=1}^{m} l_{k}^{-1}\left(R-p_{k}\right)<1$ then $\left\|\mathbf{x}_{\mathbf{p}}\right\|_{1}<1$ and if $\sum_{k=1}^{m} l_{k}^{-1}\left(R-p_{k}\right) \geq 1$ then $\left\|\mathbf{x}_{\mathbf{p}}\right\|_{1}=1$. This completes the proof.

Motivated by this example, one can consider the following payoff function

$$
L_{\varepsilon}(\mathbf{x})=\left(\varepsilon l_{1}\left(x_{1}\right), \varepsilon l_{2}\left(x_{2}\right), \cdots, \varepsilon l_{m}\left(x_{m}\right)\right) \quad \forall \varepsilon \in(-1,1)
$$

which takes on positive as well as negativen values and study dynamics of the discrete-time replicator equation given by (2.1). In this paper, we study only the Ricker model case $l_{1}(t)=l_{2}(t)=\cdots=l_{m}(t)=$ $t^{r} e^{\theta(1-t)}$ with $0 \leq \theta \leq r-\sqrt{r}$ and $r \geq 1$. The general case will be considered in the forthcoming paper [27].

A Ricker model, which was first introduced by Bill Ricker (see [26]) in 1954 in the context of stock and recruitment in fisheries, is a classic discrete population model which describes the density of individuals in the next generation as a function $f(x)=x e^{\theta(1-x)}$ of the density of individuals in the previous generation for $0<\theta \leq 1$.

We identify an evolutionary game with a smooth nonlinear payoff mapping $F: \mathbb{S}^{m-1} \rightarrow \mathbb{R}^{m}$. We assume that the nonlinear payoff mapping $F: \mathbb{S}^{m-1} \rightarrow \mathbb{R}^{m}$ is defined by the Ricker models as follows

$$
F(\mathbf{x})=\left(\varepsilon x_{1}^{r} e^{\theta\left(1-x_{1}\right)} g(\mathbf{x}), \varepsilon x_{2}^{r} e^{\theta\left(1-x_{2}\right)} g(\mathbf{x}), \cdots, \varepsilon x_{m}^{r} e^{\theta\left(1-x_{m}\right)} g(\mathbf{x})\right)
$$

where $-1<\varepsilon<1,0 \leq \theta \leq r-\sqrt{r}, r \geq 1$ are any real numbers, and $g: \mathbb{S}^{m-1} \rightarrow(0,1)$ is a smooth nonvanishing function, i.e., one has $g(\mathbf{x}) \neq 0$ for any $\mathbf{x} \in \mathbb{S}^{m-1}$. Since $g$ is a continuous function, we always have that $C_{1} \leq g(\mathbf{x}) \leq C_{2}$ for any $\mathbf{x} \in \mathbb{S}^{m-1}$ for some suitable constants $0<C_{1}<C_{2}<1$.

We now consider the discrete-time replicator equation $\mathcal{R}: \mathbb{S}^{m-1} \rightarrow \mathbb{S}^{m-1}$ defined by the nonlinear payoff mapping (3.1) as follows

$$
(\mathcal{R}(\mathbf{x}))_{k}=x_{k}\left[1+\varepsilon\left(x_{k}^{r} e^{\theta\left(1-x_{k}\right)}-\sum_{i=1}^{m} x_{i}^{r+1} e^{\theta\left(1-x_{i}\right)}\right) g(\mathbf{x})\right], \quad \forall k \in \mathbf{I}_{m} .
$$

Here, $\varepsilon \in(-1,1)$ is a controlling regime of the replicator equation. We always assume that $\varepsilon \neq 0$. We will see that the dynamics and the stability analysis of the replicator equation (3.2) will be depend on the controlling regime $\varepsilon$. Namely, if we switch $\varepsilon$ from positive to negative regime then the set of ESSs changes from one set to another one. This is a quite interesting feature of this replicator equation (3.2). This feature can be also seen in the following simple case.

Remark 3.2. If $g$ is constant, i.e., $g(\mathbf{x})=C>0$ for all $\mathbf{x} \in \mathbb{S}^{m-1}$ then we get

$$
F_{0}(\mathbf{x})=\left(\varepsilon C x_{1}^{r} e^{\theta\left(1-x_{1}\right)}, \varepsilon C x_{2}^{r} e^{\theta\left(1-x_{2}\right)}, \cdots, \varepsilon C x_{m}^{r} e^{\theta\left(1-x_{m}\right)}\right) .
$$

1. A referee pointed out that a negative case with an increasing function is equivalent to a positive case with a decreasing function which has a lot of applications. 
It is easy to check that

$$
\left(F_{0}(\mathbf{x})-F_{0}(\mathbf{y}), \mathbf{x}-\mathbf{y}\right)=\varepsilon e^{\theta} C \sum_{k=1}^{m}\left(x_{k}^{r} e^{-\theta x_{k}}-y_{k}^{r} e^{-\theta y_{k}}\right)\left(x_{k}-y_{k}\right), \quad \forall \mathbf{x}, \mathbf{y} \in \mathbb{S}^{m-1} .
$$

Since $0<\theta \leq r$ and $\mathbf{x}, \mathbf{y} \in \mathbb{S}^{m-1}$, it is clear $\left(x_{k}^{r} e^{-\theta x_{k}}-y_{k}^{r} e^{-\theta y_{k}}\right)\left(x_{k}-y_{k}\right) \geq 0$ for any $k \in \mathbf{I}_{m}$. Consequently, we have for any $\mathbf{x}, \mathbf{y} \in \mathbb{S}^{m-1}$ that if $\varepsilon>0$ then $\left(F_{0}(\mathbf{x})-F_{0}(\mathbf{y}), \mathbf{x}-\mathbf{y}\right) \geq 0$ and if $\varepsilon<0$ then $\left(F_{0}(\mathbf{x})-F_{0}(\mathbf{y}), \mathbf{x}-\mathbf{y}\right) \leq 0$.

An evolutionary game with the nonlinear payoff mapping $F: \mathbb{S}^{m-1} \rightarrow \mathbb{R}^{m}$ which satisfies the condition $(F(\mathbf{x})-F(\mathbf{y}), \mathbf{x}-\mathbf{y}) \leq 0$ for any $\mathbf{x}, \mathbf{y} \in \mathbb{S}^{m-1}$ is called a stable game. The dynamics of the continuous-time replicator equation

$$
\dot{x}_{k}=x_{k}\left(f_{k}(\mathbf{x})-\mathcal{E}_{F}(\mathbf{x}, \mathbf{x})\right), \quad \forall \mathbf{x} \in \mathbb{S}^{m-1} \text { and } k \in \mathbf{I}_{m}
$$

governed with the stable game has been studied in the paper [10] (also see [31]).

A nonlinear payoff mapping $F: \mathbb{S}^{m-1} \rightarrow \mathbb{R}^{m}$ is called monotone if one has that $(F(\mathbf{x})-F(\mathbf{y}), \mathbf{x}-\mathbf{y}) \geq 0$ for any $\mathbf{x}, \mathbf{y} \in \mathbb{S}^{m-1}$. The dynamics of the discrete-time replicator equation

$$
x_{k}^{\prime}=x_{k}\left(1+f_{k}(\mathbf{x})-\mathcal{E}_{F}(\mathbf{x}, \mathbf{x})\right), \quad \forall \mathbf{x} \in \mathbb{S}^{m-1} \text { and } k \in \mathbf{I}_{m}
$$

has been studied in the paper [9].

Hence, if $g$ is constant then the nonlinear payoff mapping $F: \mathbb{S}^{m-1} \rightarrow \mathbb{R}^{m}$ given by (3.1) is either monotone or stable depending on the controlling regime $\varepsilon \in(-1,1)$. In general, if $g$ is not constant then the nonlinear payoff mapping $F: \mathbb{S}^{m-1} \rightarrow \mathbb{R}^{m}$ given by (3.1) is neither monotone nor stable. In this paper, we are aiming to study the dynamics of the discrete-time replicator equation $\mathcal{R}: \mathbb{S}^{m-1} \rightarrow \mathbb{S}^{m-1}$ given by (3.2) for any given non-constant function $g$.

We now describe the set NE $(F)$ (resp. SNE $(F)$ ) of all Nash equilibria (resp. strict Nash equilibria) of the nonlinear payoff mapping $F: \mathbb{S}^{m-1} \rightarrow \mathbb{R}^{m}$ given by (3.1) and the set $\mathbf{F i x}(\mathcal{R})$ of fixed (rest) points of the discrete-time replicator equation $\mathcal{R}: \mathbb{S}^{m-1} \rightarrow \mathbb{S}^{m-1}$ given by (3.2).

Proposition 3.3. Let $\mathbf{e}_{\alpha}:=\frac{1}{|\alpha|} \sum_{i \in \alpha} \mathbf{e}_{i}$ be the center of the face $\mathbb{S}^{|\alpha|-1}$ for $\alpha \subset \mathbf{I}_{m}$. Let $\mathbf{c}:=\mathbf{e}_{\mathbf{I}_{m}}=\left(\frac{1}{m}, \cdots, \frac{1}{m}\right)$ be the center of the simplex $\mathbb{S}^{m-1}$. Then the following statements hold true:

(i) For any nonzero $\varepsilon \in(-1,1)$, one has $\mathbf{F i x}(\mathcal{R})=\bigcup_{\alpha \subset \mathbf{I}_{m}}\left\{\mathbf{e}_{\alpha}\right\}$;

(ii) For any $\varepsilon \in(0,1)$, one has $\mathbf{S N E}(F)=\left\{\mathbf{e}_{k}\right\}_{k=1}^{n} \subset \mathbf{N E}(F)=\mathbf{F i x}(\mathcal{R})$;

(iii) For any $\varepsilon \in(-1,0)$, one has $\mathbf{S N E}(F)=\emptyset$ and $\mathbf{N E}(F)=\{\mathbf{c}\} \subset \mathbf{F i x}(\mathcal{R})$.

Proof. (i) We first show $\operatorname{Fix}(\mathcal{R})=\bigcup_{\alpha \subset \mathbf{I}_{m}}\left\{\mathbf{e}_{\alpha}\right\}$. It is obvious that $\bigcup_{\alpha \subset \mathbf{I}_{m}}\left\{\mathbf{e}_{\alpha}\right\} \subset \mathbf{F i x}(\mathcal{R})$. Let $\mathbf{x} \in \mathbf{F i x}(\mathcal{R})$ be a fixed point. We set $\alpha:=\operatorname{supp}(\mathbf{x})$. Since $\varepsilon \neq 0$ and $g(\mathbf{x})>0$ for any $\mathbf{x} \in \mathbb{S}^{m-1}$, it follows from (3.2)

$$
x_{k}^{r} e^{\theta\left(1-x_{k}\right)}=\sum_{i \in \alpha} x_{i}^{r+1} e^{\theta\left(1-x_{i}\right)}, \quad \forall k \in \alpha .
$$

This means that $x_{k_{1}}^{r} e^{\theta\left(1-x_{k_{1}}\right)}=x_{k_{2}}^{r} e^{\theta\left(1-x_{k_{2}}\right)}$ for any distinct $k_{1}, k_{2} \in \alpha$ or equivalently $x_{k_{1}}=x_{k_{2}}$ for any $k_{1}, k_{2} \in \alpha$. Hence, we get $\mathbf{x}=\mathbf{e}_{\alpha}$. This shows $\boldsymbol{F i x}(\mathcal{R})=\bigcup_{\alpha \subset \mathbf{I}_{m}}\left\{\mathbf{e}_{\alpha}\right\}$. 
(ii) Let $\varepsilon>0$. We first show $\mathbf{N E}(F)=\bigcup_{\alpha \subset \mathbf{I}_{m}}\left\{\mathbf{e}_{\alpha}\right\}$. Since for any $\mathbf{y} \in \mathbb{S}^{m-1}$ and $\alpha \subset \mathbf{I}_{m}$

$$
\mathcal{E}_{F}\left(\mathbf{e}_{\alpha}, \mathbf{e}_{\alpha}\right)=\varepsilon \frac{e^{\theta\left(1-|\alpha|^{-1}\right)}}{|\alpha|^{r}} g\left(\mathbf{e}_{\alpha}\right) \geq \varepsilon\left(\sum_{i \in \alpha} y_{i}\right) \frac{e^{\theta\left(1-|\alpha|^{-1}\right)}}{|\alpha|^{r}} g\left(\mathbf{e}_{\alpha}\right)=\mathcal{E}_{F}\left(\mathbf{y}, \mathbf{e}_{\alpha}\right),
$$

we get $\bigcup_{\alpha \subset \mathbf{I}_{m}}\left\{\mathbf{e}_{\alpha}\right\} \subset \mathbf{N E}(F)$. Let $\mathbf{x} \in \mathbf{N E}(F)$ and $\alpha:=\operatorname{supp}(\mathbf{x})$. We have for $\mathbf{z} \in \mathbb{S}^{m-1}$

$$
\varepsilon\left(\sum_{i \in \alpha} z_{i}\right) \min _{i \in \alpha}\left\{x_{i}^{r} e^{\theta\left(1-x_{i}\right)}\right\} g(\mathbf{x}) \leq \mathcal{E}_{F}(\mathbf{z}, \mathbf{x}) \leq \varepsilon\left(\sum_{i \in \alpha} z_{i}\right) \max _{i \in \alpha}\left\{x_{i}^{r} e^{\theta\left(1-x_{i}\right)}\right\} g(\mathbf{x}) .
$$

Since $\mathbf{x} \in \mathbf{N E}(F)$, we must have

$$
\mathcal{E}_{F}(\mathbf{x}, \mathbf{x})=\max _{\mathbf{z} \in \mathbb{S}^{m-1}} \mathcal{E}_{F}(\mathbf{z}, \mathbf{x})=\varepsilon \max _{i \in \alpha}\left\{x_{i}^{r} e^{\theta\left(1-x_{i}\right)}\right\} g(\mathbf{x})
$$

On the other hand, we have

$$
\mathcal{E}_{F}(\mathbf{x}, \mathbf{x})=\varepsilon\left(\sum_{i \in \alpha} x_{i}^{r+1} e^{\theta\left(1-x_{i}\right)}\right) g(\mathbf{x}) \leq \varepsilon \max _{i \in \alpha}\left\{x_{i}^{r} e^{\theta\left(1-x_{i}\right)}\right\} g(\mathbf{x}) .
$$

Consequently, we obtain $x_{k}^{r} e^{\theta\left(1-x_{k}\right)}=\max _{i \in \alpha}\left\{x_{i}^{r} e^{\theta\left(1-x_{i}\right)}\right\}$ for any $k \in \alpha$. This means that $x_{k_{1}}=x_{k_{2}}$ for any $k_{1}, k_{2} \in \alpha$ or $\mathbf{x}=\mathbf{e}_{\alpha}$. This shows $\mathbf{N E}(F)=\bigcup_{\alpha \subset \mathbf{I}_{m}}\left\{\mathbf{e}_{\alpha}\right\}$.

We now show $\left\{\mathbf{e}_{k}\right\}_{k=1}^{n} \subset \mathbf{S N E}(F)$, i.e., the strategy $\mathbf{e}_{k}$ is the unique best reply to itself, $\operatorname{BR}\left(\mathbf{e}_{k}\right)=\left\{\mathbf{e}_{k}\right\}$ for all $k \in \mathbf{I}_{m}$. Indeed, for all $\mathbf{y} \in \mathbb{S}^{m-1} \backslash\left\{\mathbf{e}_{k}\right\}$ we have that

$$
\mathcal{E}_{F}\left(\mathbf{e}_{k}, \mathbf{e}_{k}\right)=\varepsilon 1^{r+1} e^{\theta(1-1)} g\left(\mathbf{e}_{k}\right)>\varepsilon y_{k} 1^{r} e^{\theta(1-1)} g\left(\mathbf{e}_{k}\right)=\mathcal{E}_{F}\left(\mathbf{y}, \mathbf{e}_{k}\right) .
$$

On the other hand, we have for any $\alpha \subset \mathbf{I}_{m}$ with $|\alpha|>1$ and $k \in \alpha$

$$
\mathcal{E}_{F}\left(\mathbf{e}_{\alpha}, \mathbf{e}_{\alpha}\right)=\varepsilon\left(\sum_{i \in \alpha} \frac{1}{|\alpha|}\right) \frac{e^{\theta\left(1-|\alpha|^{-1}\right)}}{|\alpha|^{r}} g\left(\mathbf{e}_{\alpha}\right)=\varepsilon \cdot 1 \cdot \frac{\left.e^{\theta\left(1-|\alpha|^{-1}\right.}\right)}{|\alpha|^{r}} g\left(\mathbf{e}_{\alpha}\right)=\mathcal{E}_{F}\left(\mathbf{e}_{k}, \mathbf{e}_{\alpha}\right) .
$$

This means that the Nash equilibrium $\mathbf{e}_{\alpha}$ for any $\alpha \subset \mathbf{I}_{m}$ with $|\alpha|>1$ is not the strict Nash equilibrium. Consequently, the strict Nash equilibria are only vertices of the simplex, i.e., $\mathbf{S N E}(F)=\left\{\mathbf{e}_{k}\right\}_{k=1}^{n}$.

(iii) Let $\varepsilon<0$. We first show $\mathbf{N E}(F)=\{\mathbf{c}\}$. It is obvious that $\mathbf{c} \in \mathbf{N E}(F)$.

Let $\mathbf{x} \in \mathbf{N E}(F)$ and $\alpha:=\operatorname{supp}(\mathbf{x})$. We then want to prove that $\alpha=\mathbf{I}_{m}$. We assume the contrary, i.e., $\alpha \neq \mathbf{I}_{m}$. Let $k \in \mathbf{I}_{m} \backslash \alpha$ (since $\left.\mathbf{I}_{m} \backslash \alpha \neq \emptyset\right)$. Since $\varepsilon<0, g(\mathbf{x})>0$, and $\mathbf{x} \in \mathbf{N E}(F)$, we face the contradiction

$$
0=\mathcal{E}_{F}\left(\mathbf{e}_{k}, \mathbf{x}\right) \leq \mathcal{E}_{F}(\mathbf{x}, \mathbf{x})=\varepsilon\left(\sum_{i \in \alpha} x_{i}^{r+1} e^{\theta\left(1-x_{i}\right)}\right) g(\mathbf{x})<0 .
$$

This shows $\alpha=\mathbf{I}_{m}$. In this case, we have for any $\mathbf{z} \in \mathbb{S}^{m-1}$

$$
\varepsilon \max _{i \in \mathbf{I}_{m}}\left\{x_{i}^{r} e^{\theta\left(1-x_{i}\right)}\right\} g(\mathbf{x}) \leq \mathcal{E}_{F}(\mathbf{z}, \mathbf{x}) \leq \varepsilon \min _{i \in \mathbf{I}_{m}}\left\{x_{i}^{r} e^{\theta\left(1-x_{i}\right)}\right\} g(\mathbf{x}) .
$$


Since $\mathbf{x} \in \mathbf{N E}(F)$, we must have

$$
\mathcal{E}_{F}(\mathbf{x}, \mathbf{x})=\max _{\mathbf{z} \in \mathbb{S}^{m-1}} \mathcal{E}_{F}(\mathbf{z}, \mathbf{x})=\varepsilon \min _{i \in \mathbf{I}_{m}}\left\{x_{i}^{r} e^{\theta\left(1-x_{i}\right)}\right\} g(\mathbf{x}) .
$$

On the other hand, we have

$$
\mathcal{E}_{F}(\mathbf{x}, \mathbf{x})=\varepsilon\left(\sum_{i=1}^{m} x_{i}^{r+1} e^{\theta\left(1-x_{i}\right)}\right) g(\mathbf{x}) \leq \varepsilon \min _{i \in \mathbf{I}_{m}}\left\{x_{i}^{r} e^{\theta\left(1-x_{i}\right)}\right\} g(\mathbf{x}) .
$$

Consequently, we obtain $x_{k}^{r} e^{\theta\left(1-x_{k}\right)}=\min _{i \in \mathbf{I}_{m}}\left\{x_{i}^{r} e^{\theta\left(1-x_{i}\right)}\right\}$ for any $k \in \mathbf{I}_{m}$. This means that $x_{k_{1}}=x_{k_{2}}$ for any $k_{1}, k_{2} \in \mathbf{I}_{m}$ or $\mathbf{x}=\mathbf{c}$. This shows $\mathbf{N E}(F)=\{\mathbf{c}\}$.

We now show $\mathbf{S N E}(F)=\emptyset$, i.e, the center $\mathbf{c}$ of the simplex $\mathbb{S}^{m-1}$ which is the unique Nash equilibrium is not a strict Nash equilibrium. Namely, we show $\operatorname{BR}(\mathbf{c})=\mathbb{S}^{m-1}$. Indeed, for all $\mathbf{y} \in \mathbb{S}^{m-1}$ we have that

$$
\mathcal{E}_{F}(\mathbf{c}, \mathbf{c})=\varepsilon\left(\sum_{i=1}^{m} \frac{1}{m}\right) \frac{e^{\theta\left(1-m^{-1}\right)}}{m^{r}} g(\mathbf{c})=\varepsilon\left(\sum_{i=1}^{m} y_{i}\right) \frac{e^{\theta\left(1-m^{-1}\right)}}{m^{r}} g(\mathbf{c})=\mathcal{E}_{F}(\mathbf{y}, \mathbf{c}) .
$$

In the next sections, we will describe the set $\operatorname{ESS}(F)$ of all local ESSs of the nonlinear payoff mapping $F: \mathbb{S}^{m-1} \rightarrow \mathbb{R}^{m}$ and study the dynamics (stability analysis) of the discrete-time replicator equation $\mathcal{R}: \mathbb{S}^{m-1} \rightarrow \mathbb{S}^{m-1}$.

\section{The regime $\varepsilon \in(0,1)$}

In this section, we always assume $\varepsilon \in(0,1)$. We first describe the set $\operatorname{ESS}(F)$ of all local ESSs of the nonlinear payoff mapping $F: \mathbb{S}^{m-1} \rightarrow \mathbb{R}^{m}$ given by (3.1).

Proposition 4.1. We have $\operatorname{ESS}(F)=\left\{\mathbf{e}_{1}, \mathbf{e}_{2}, \cdots, \mathbf{e}_{m}\right\}$ where $\mathbf{e}_{i}$ is the vertex of the simplex $\mathbb{S}^{m-1}$ for $i \in \mathbf{I}_{m}$.

Proof. According to Definition 2.2 (see item (i)), we have $\mathbf{E S S}(F) \subset \mathbf{N E}(F)$. Let us first show $\left\{\mathbf{e}_{1}, \mathbf{e}_{2}, \cdots, \mathbf{e}_{m}\right\} \subset \mathbf{E S S}(F)$. It order to accomplish it, we want to show that there is a sufficiently small neighborhood $U\left(\mathbf{e}_{k}\right) \subset \mathbb{S}^{m-1}$ of the vertex $\mathbf{e}_{k}$ for $k \in \mathbf{I}_{m}$ such that $\mathcal{E}_{F}\left(\mathbf{e}_{k}, \mathbf{y}\right)>\mathcal{E}_{F}(\mathbf{y}, \mathbf{y})$ for all $\mathbf{y} \in U\left(\mathbf{e}_{k}\right) \backslash\left\{\mathbf{e}_{k}\right\}$. Since $U\left(\mathbf{e}_{k}\right) \subset \mathbb{S}^{m-1}$ is sufficiently small, we have MaxInd $(\mathbf{y})=\{k\}$ and $\operatorname{supp}(\mathbf{y}) \backslash\{k\} \neq \emptyset$ for all $\mathbf{y} \in U\left(\mathbf{e}_{k}\right) \backslash\left\{\mathbf{e}_{k}\right\}$. Consequently, we get for all $\mathbf{y} \in U\left(\mathbf{e}_{k}\right) \backslash\left\{\mathbf{e}_{k}\right\}$

$$
\begin{aligned}
\mathcal{E}_{F}(\mathbf{y}, \mathbf{y}) & =\varepsilon\left(\sum_{i \in \operatorname{supp}(\mathbf{y})} y_{i}^{r+1} e^{\theta\left(1-y_{i}\right)}\right) g(\mathbf{y}) \\
& <\varepsilon \max _{i \in \operatorname{supp}(\mathbf{y})}\left\{y_{i}^{r} e^{\theta\left(1-y_{i}\right)}\right\} g(\mathbf{y})=\varepsilon\left(y_{k}^{r} e^{\theta\left(1-y_{k}\right)}\right) g(\mathbf{y})=\mathcal{E}_{F}\left(\mathbf{e}_{k}, \mathbf{y}\right) .
\end{aligned}
$$

This shows $\left\{\mathbf{e}_{1}, \mathbf{e}_{2}, \cdots, \mathbf{e}_{m}\right\} \subset \mathbf{E S S}(F)$.

We now show $\mathbf{E S S}(F) \cap \mathbf{N E}(F)=\left\{\mathbf{e}_{1}, \mathbf{e}_{2}, \cdots, \mathbf{e}_{m}\right\}$. Let $\mathbf{e}_{\alpha} \in \mathbf{N E}(F)$ with $|\alpha| \geq 2$ be any Nash equilibrium. Let $\alpha:=\left\{i_{1}, i_{2}, \cdots, i_{|\alpha|}\right\}$ such that $i_{1}<i_{2}<\cdots<i_{|\alpha|}$. We want to show that for any sufficiently small neighborhood $U\left(\mathbf{e}_{\alpha}\right) \subset \mathbb{S}^{m-1}$ of the point $\mathbf{e}_{\alpha}$ there always exists $\overline{\mathbf{y}} \in U\left(\mathbf{e}_{\alpha}\right) \backslash\left\{\mathbf{e}_{\alpha}\right\}$ such that $\mathcal{E}_{F}\left(\mathbf{e}_{\alpha}, \overline{\mathbf{y}}\right)<\mathcal{E}_{F}(\overline{\mathbf{y}}, \overline{\mathbf{y}})$. Let $\delta>0$ be any sufficiently small number and $\left\|\overline{\mathbf{y}}-\mathbf{e}_{\alpha}\right\|_{1}<\delta$ such that 
$\operatorname{supp}(\overline{\mathbf{y}})=\alpha$ and $\bar{y}_{i_{1}}<\bar{y}_{i_{2}}<\cdots<\bar{y}_{i_{|\alpha|}}$ (it is always possible to choose such $\overline{\mathbf{y}} \in U\left(\mathbf{e}_{\alpha}\right)$ for any $\left.\delta>0\right)$. Since $\bar{y}_{i_{1}}^{r} e^{\theta\left(1-\bar{y}_{i_{1}}\right)}<\bar{y}_{i_{2}}^{r} e^{\theta\left(1-\bar{y}_{i_{2}}\right)}<\cdots<\bar{y}_{i_{|\alpha|}}^{r} e^{\theta\left(1-\bar{y}_{i \alpha \mid}\right)}$, it follows from Chebyshev's sum inequality (see [19])

$$
\mathcal{E}_{F}\left(\mathbf{e}_{\alpha}, \overline{\mathbf{y}}\right)=\varepsilon\left(\frac{1}{|\alpha|} \sum_{j=1}^{|\alpha|} \bar{y}_{i_{j}}^{r} e^{\theta\left(1-\bar{y}_{i_{j}}\right)}\right) g(\overline{\mathbf{y}})<\varepsilon\left(\sum_{j=1}^{|\alpha|} \bar{y}_{i_{j}}^{r+1} e^{\theta\left(1-\bar{y}_{i_{j}}\right)}\right) g(\overline{\mathbf{y}})=\mathcal{E}_{F}(\overline{\mathbf{y}}, \overline{\mathbf{y}}) .
$$

It means that $\mathbf{e}_{\alpha} \in \mathbf{N E}(F)(|\alpha| \geq 2)$ is not a local ESS. This completes the proof.

We now study the dynamics (the stability analysis) of the discrete-time replicator equation given by (3.2) for $\varepsilon>0$ by means of the Lyapunov function.

Theorem 4.2. Let $\mathcal{R}: \mathbb{S}^{m-1} \rightarrow \mathbb{S}^{m-1}$ be the discrete-time replicator equation given by (3.2) and $\varepsilon \in(0,1)$. Then the following statements hold true:

(i) The functions $\mathcal{M}_{k}(\mathbf{x}):=\max _{i \in \mathbf{I}_{m}}\left\{x_{i}\right\}-x_{k}$ for all $k \in \mathbf{I}_{m}$ are the increasing Lyapunov functions for $\mathcal{R}: \operatorname{int} \mathbb{S}^{m-1} \rightarrow \operatorname{int} \mathbb{S}^{m-1}$

(ii) The functions $\mathcal{M}_{\alpha, k}(\mathbf{x}):=\max _{i \in \alpha}\left\{x_{i}\right\}-x_{k}$ for all $k \in \alpha$ are the increasing Lyapunov functions for $\mathcal{R}: \operatorname{int} \mathbb{S}^{|\alpha|-1} \rightarrow \operatorname{int} \mathbb{S}^{|\alpha|-1}$

(iii) The trajectory of the replicator equation $\mathcal{R}: \mathbb{S}^{m-1} \rightarrow \mathbb{S}^{m-1}$ starting from any initial point $\mathbf{x} \in \mathbb{S}^{m-1}$ converges to the center of the face $\mathbb{S}^{|\operatorname{Max} I n d(\mathbf{x})|-1}$.

Proof. (i) We show that $\mathcal{M}_{k}(\mathbf{x})$ for $k \in \mathbf{I}_{m}$ is the increasing Lyapunov function along the trajectory of the operator $\mathcal{R}: \operatorname{int} \mathbb{S}^{m-1} \rightarrow \operatorname{int} \mathbb{S}^{m-1}$. Let $\mathbf{x} \in \operatorname{int} \mathbb{S}^{m-1}$.

It follows from (3.2) for any $k, t \in \mathbf{I}_{m}$ that if $x_{t}=x_{k}$ then $(\mathcal{R}(\mathbf{x}))_{t}=(\mathcal{R}(\mathbf{x}))_{k}$ and if $x_{t} \neq x_{k}$ then

$$
\begin{aligned}
(\mathcal{R}(\mathbf{x}))_{t} & -(\mathcal{R}(\mathbf{x}))_{k} \\
& =\left(x_{t}-x_{k}\right)\left[1+\varepsilon\left(\frac{x_{t}^{r+1} e^{\theta\left(1-x_{t}\right)}-x_{k}^{r+1} e^{\theta\left(1-x_{k}\right)}}{x_{t}-x_{k}}-\sum_{i=1}^{m} x_{i}^{r+1} e^{\theta\left(1-x_{i}\right)}\right) g(\mathbf{x})\right]
\end{aligned}
$$

which yields the following equality

$$
\begin{aligned}
(\mathcal{R}(\mathbf{x}))_{t}-(\mathcal{R}(\mathbf{x}))_{k} & =\left(x_{t}-x_{k}\right)(1-\varepsilon g(\mathbf{x}))+ \\
& +\varepsilon\left(x_{t}-x_{k}\right)\left[\frac{x_{t}^{r+1} e^{\theta\left(1-x_{t}\right)}-x_{k}^{r+1} e^{\theta\left(1-x_{k}\right)}}{x_{t}-x_{k}}+\sum_{i=1}^{m} x_{i}\left(1-x_{i}^{r} e^{\theta\left(1-x_{i}\right)}\right)\right] g(\mathbf{x})
\end{aligned}
$$

Since $0<\varepsilon g(\mathbf{x})<1,0 \leq x_{i}^{r} e^{\theta\left(1-x_{i}\right)} \leq 1$ for all $i \in \mathbf{I}_{m}$ and

$$
\left(x_{t}^{r+1} e^{\theta\left(1-x_{t}\right)}-x_{k}^{r+1} e^{\theta\left(1-x_{k}\right)}\right)\left(x_{t}-x_{k}\right) \geq 0, \quad \forall \mathbf{x} \in \operatorname{int} \mathbb{S}^{m-1},
$$

we obtain $\operatorname{Sign}\left((\mathcal{R}(\mathbf{x}))_{t}-(\mathcal{R}(\mathbf{x}))_{k}\right)=\operatorname{Sign}\left(x_{t}-x_{k}\right)$ for any $k, t \in \mathbf{I}_{m}$ and

$$
\operatorname{Max} \operatorname{Ind}(\mathcal{R}(\mathbf{x}))=\operatorname{Max} \operatorname{Ind}(\mathbf{x}), \quad \forall \mathbf{x} \in \operatorname{int} \mathbb{S}^{m-1} .
$$


Moreover, if $t \in \operatorname{Max} \operatorname{Ind}(\mathcal{R}(\mathbf{x}))=\operatorname{Max} \operatorname{Ind}(\mathbf{x})$ then for all $k \in \mathbf{I}_{m}$

$$
\begin{aligned}
(\mathcal{R}(\mathbf{x}))_{t}-(\mathcal{R}(\mathbf{x}))_{k} & =\left(x_{t}-x_{k}\right)+ \\
& +\varepsilon\left(x_{t}-x_{k}\right)\left(\frac{x_{k}\left(x_{t}^{r} e^{\theta\left(1-x_{t}\right)}-x_{k}^{r} e^{\theta\left(1-x_{k}\right)}\right)}{x_{t}-x_{k}}+\sum_{i=1}^{m} x_{i}\left(x_{t}^{r} e^{\theta\left(1-x_{t}\right)}-x_{i}^{r} e^{\theta\left(1-x_{i}\right)}\right)\right) g(\mathbf{x})
\end{aligned}
$$

This means $\mathcal{M}_{k}(\mathcal{R}(\mathbf{x})) \geq \mathcal{M}_{k}(\mathbf{x})$ for all $k \in \mathbf{I}_{m}$.

By repeating this process, we get for all $k \in \mathbf{I}_{m}, n \in \mathbb{N}$, and $\mathbf{x} \in \operatorname{int} \mathbb{S}^{m-1}$ that

$$
\operatorname{Max} \operatorname{Ind}\left(\mathcal{R}^{(n)}(\mathbf{x})\right)=\operatorname{Max} \operatorname{Ind}(\mathbf{x}), \quad \mathcal{M}_{k}\left(\mathcal{R}^{(n+1)}(\mathbf{x})\right) \geq \mathcal{M}_{k}\left(\mathcal{R}^{(n)}(\mathbf{x})\right) .
$$

This shows that $\mathcal{M}_{k}(\mathbf{x})$ for all $k \in \mathbf{I}_{m}$ is the Lyapunov function over the set int $\mathbb{S}^{m-1}$.

The part (ii) can be similarly proved. Its proof is omitted here.

We now prove the part (iii). Without loss of generality, we may assume that $\mathbf{x} \in$ int $\mathbb{S}^{m-1}$. Otherwise, we choose the suitable Lyapunov function given in the part (ii) depending on an initial point $\mathbf{x} \in \mathbb{S}^{m-1}$. We fix $t_{0} \in \operatorname{Max} \operatorname{lnd}(\mathbf{x})=\operatorname{Max} \operatorname{lnd}\left(\mathcal{R}^{(n)}(\mathbf{x})\right)$.

Since the sequence $\left\{\mathcal{M}_{k}\left(\mathcal{R}^{(n)}(\mathbf{x})\right)\right\}_{n=0}^{\infty}$ where

$$
\mathcal{M}_{k}\left(\mathcal{R}^{(n)}(\mathbf{x})\right)=\left(\mathcal{R}^{(n)}(\mathbf{x})\right)_{t_{0}}-\left(\mathcal{R}^{(n)}(\mathbf{x})\right)_{k}
$$

is convergent for each $k \in \mathbf{I}_{m}$, the sequence $\left\{\left(\mathcal{R}^{(n)}(\mathbf{x})\right)_{t_{0}}\right\}_{n=0}^{\infty}$ where

$$
\left(\mathcal{R}^{(n)}(\mathbf{x})\right)_{t_{0}}=\frac{1}{m}\left(1+\sum_{k=1}^{m} \mathcal{M}_{k}\left(\mathcal{R}^{(n)}(\mathbf{x})\right)\right)
$$

is also convergent. Hence, the sequence $\left\{\left(\mathcal{R}^{(n)}(\mathbf{x})\right)_{k}\right\}_{n=0}^{\infty}$ for each $k \in \mathbf{I}_{m}$ where

$$
\left(\mathcal{R}^{(n)}(\mathbf{x})\right)_{k}=\left(\mathcal{R}^{(n)}(\mathbf{x})\right)_{t_{0}}-\mathcal{M}_{k}\left(\mathcal{R}^{(n)}(\mathbf{x})\right)
$$

is also convergent. This means that the trajectory $\left\{\mathcal{R}^{(n)}(\mathbf{x})\right\}_{n=0}^{\infty}$ is convergent and its omega limiting point is some fixed (rest) point $\mathbf{x}^{*}$. We now want to show that

$$
\operatorname{Max} \operatorname{lnd}\left(\mathbf{x}^{*}\right)=\operatorname{Max} \operatorname{lnd}(\mathbf{x})
$$

Since MaxInd $(\mathbf{x})=\operatorname{MaxInd}\left(\mathcal{R}^{(n)}(\mathbf{x})\right)$ and $\mathcal{M}_{k}\left(\mathcal{R}^{(n+1)}(\mathbf{x})\right) \geq \mathcal{M}_{k}\left(\mathcal{R}^{(n)}(\mathbf{x})\right)$ for any $n \in \mathbb{N}$ and $k \in \mathbf{I}_{m}$, we have that $\operatorname{Max} \operatorname{Ind}\left(\mathbf{x}^{*}\right) \supset \operatorname{Max} \operatorname{lnd}(\mathbf{x})$.

We now show MaxInd $\left(\mathbf{x}^{*}\right) \subset \operatorname{Max} \operatorname{Ind}(\mathbf{x})$. Indeed, if $k_{0} \in \operatorname{MaxInd}\left(\mathbf{x}^{*}\right)$ then we get that

$$
0 \leq \mathcal{M}_{k_{0}}(\mathbf{x}) \leq \mathcal{M}_{k_{0}}(\mathcal{R}(\mathbf{x})) \leq \cdots \mathcal{M}_{k_{0}}\left(\mathcal{R}^{(n)}(\mathbf{x})\right) \leq \cdots \leq \mathcal{M}_{k_{0}}\left(\mathbf{x}^{*}\right)=0
$$

This means $\mathcal{M}_{k_{0}}(\mathbf{x})=0$ or $k_{0} \in \operatorname{Max} I n d(\mathbf{x})$. Hence, we prove MaxInd( $\left.\mathbf{x}^{*}\right)=\operatorname{Max} \operatorname{lnd}(\mathbf{x})$. Consequently, due to Proposition 3.3 , among the centers of all faces of the simplex the only fixed point $\mathbf{x}^{*}$ which satisfies the property MaxInd $\left(\mathbf{x}^{*}\right)=\operatorname{Max} \operatorname{Ind}(\mathbf{x})$ is the center of the face $\mathbb{S}^{|\operatorname{Max} I n d(\mathbf{x})|-1}$. This completes the proof. 
5. The regime $\varepsilon \in(-1,0)$

In this section, we always assume $\varepsilon \in(-1,0)$. We first describe the set $\operatorname{ESS}(F)$ of all local ESSs of the nonlinear payoff mapping $F: \mathbb{S}^{m-1} \rightarrow \mathbb{R}^{m}$ given by $(\underline{3.1})$.

Proposition 5.1. We have $\operatorname{ESS}(F)=\{\mathbf{c}\}$ where $\mathbf{c}$ is the center of the simplex $\mathbb{S}^{m-1}$.

Proof. According to Definition 2.2 , we have $\mathbf{E S S}(F) \subset \mathbf{N E}(F)=\{\mathbf{c}\}$. Let us show $\mathbf{c} \in \mathbf{E S S}(F)$. Let $U(\mathbf{c}) \subset \mathbb{S}^{m-1}$ be a sufficiently small neighborhood of the center c of the simplex $\mathbb{S}^{m-1}$. Since $\varepsilon<0$, the vectors $\mathbf{y}$ and $F(\mathbf{y})$ are the same ordered, i.e., if $y_{i}>y_{j}\left(\right.$ resp. $\left.y_{i}<y_{j}\right)$ then $(F(\mathbf{y}))_{i}>(F(\mathbf{y}))_{j}($ resp. $\left.(F(\mathbf{y}))_{i}<(F(\mathbf{y}))_{j}\right)$, it then follows from Chebyshev's sum inequality (see [19])

$$
\mathcal{E}_{F}(\mathbf{c}, \mathbf{y})=\varepsilon\left(\frac{1}{m} \sum_{i=1}^{m} y_{i}^{r} e^{\theta\left(1-y_{i}\right)}\right) g(\mathbf{y})>\varepsilon\left(\sum_{i=1}^{m} y_{i}^{r+1} e^{\theta\left(1-y_{i}\right)}\right) g(\mathbf{y})=\mathcal{E}_{F}(\mathbf{y}, \mathbf{y}) .
$$

for any $\mathbf{y} \in U(\mathbf{c}) \backslash\{\mathbf{c}\}$ (for our case, the equality in Chebyshev's sum inequality holds only for $\mathbf{y}=\mathbf{c}$ ). It means $\mathbf{c} \in \mathbf{E S S}(F)$. This completes the proof.

We now study the dynamics (the stability analysis) of the discrete-time replicator equation given by (3.2) for $\varepsilon<0$ by means of the Lyapunov function.

We define the following constants

$$
K:=\sqrt{r+1}\left(\frac{r+1-\sqrt{r+1}}{\theta}\right)^{r} e^{\theta+\sqrt{r+1}-r-1}, \quad \mu:=\max _{\mathbf{x} \in \mathbb{S}^{m-1}} g(\mathbf{x}) \cdot \max \{1+r-\theta ; K\}
$$

Theorem 5.2. Let $\mathcal{R}: \mathbb{S}^{m-1} \rightarrow \mathbb{S}^{m-1}$ be the discrete-time replicator equation given by (3.2) and $\varepsilon \in$ $\left(-\frac{1}{\mu}, 0\right) \cap(-1,0)$. Then the following statements hold true:

(i) The functions $\mathcal{M}_{k}(\mathbf{x}):=\max _{i \in \mathbf{I}_{m}}\left\{x_{i}\right\}-x_{k}$ for all $k \in \mathbf{I}_{m}$ are the decreasing Lyapunov functions for $\mathcal{R}: \operatorname{int} \mathbb{S}^{m-1} \rightarrow \operatorname{int} \mathbb{S}^{m-1}$

(ii) The functions $\mathcal{M}_{\alpha, k}(\mathbf{x}):=\max _{i \in \alpha}\left\{x_{i}\right\}-x_{k}$ for all $k \in \alpha$ are the decreasing Lyapunov functions for $\mathcal{R}: \operatorname{int} \mathbb{S}^{|\alpha|-1} \rightarrow \operatorname{int} \mathbb{S}^{|\alpha|-1}$

(iii) The trajectory of the replicator equation $\mathcal{R}: \mathbb{S}^{m-1} \rightarrow \mathbb{S}^{m-1}$ starting from any initial point $\mathbf{x} \in \mathbb{S}^{m-1}$ converges to the center of the face $\mathbb{S}^{|\operatorname{supp}(\mathbf{x})|-1}$.

Proof. (i) We show that $\mathcal{M}_{k}(\mathbf{x})$ for $k \in \mathbf{I}_{m}$ is the decreasing Lyapunov function along the trajectory of the operator $\mathcal{R}:$ int $\mathbb{S}^{m-1} \rightarrow \operatorname{int} \mathbb{S}^{m-1}$. Let $\mathbf{x} \in \operatorname{int} \mathbb{S}^{m-1}$.

It follows from (3.2) for any $k, t \in \mathbf{I}_{m}$ that if $x_{t}=x_{k}$ then $(\mathcal{R}(\mathbf{x}))_{t}=(\mathcal{R}(\mathbf{x}))_{k}$ and if $x_{t} \neq x_{k}$ then

$$
\begin{aligned}
(\mathcal{R}(\mathbf{x}))_{t} & -(\mathcal{R}(\mathbf{x}))_{k} \\
& =\left(x_{t}-x_{k}\right)\left[1+\varepsilon\left(\frac{x_{t}^{r+1} e^{\theta\left(1-x_{t}\right)}-x_{k}^{r+1} e^{\theta\left(1-x_{k}\right)}}{x_{t}-x_{k}}-\sum_{i=1}^{m} x_{i}^{r+1} e^{\theta\left(1-x_{i}\right)}\right) g(\mathbf{x})\right]
\end{aligned}
$$


Since $-\frac{1}{\mu}<\varepsilon<0$ and for all $t, k \in \mathbf{I}_{m}$

$$
\left|\frac{x_{t}^{r+1} e^{\theta\left(1-x_{t}\right)}-x_{k}^{r+1} e^{\theta\left(1-x_{k}\right)}}{x_{t}-x_{k}}\right| \leq \max _{x \in[0,1]} \frac{d}{d x}\left(x^{r+1} e^{\theta(1-x)}\right)=\max \{1+r-\theta ; K\},
$$

we obtain $\operatorname{Sign}\left((\mathcal{R}(\mathbf{x}))_{t}-(\mathcal{R}(\mathbf{x}))_{k}\right)=\operatorname{Sign}\left(x_{t}-x_{k}\right)$ for any $k, t \in \mathbf{I}_{m}$ and

$$
\operatorname{Max} \operatorname{Ind}(\mathcal{R}(\mathbf{x}))=\operatorname{Max} \operatorname{Ind}(\mathbf{x}), \quad \forall \mathbf{x} \in \operatorname{int} \mathbb{S}^{m-1} .
$$

Moreover, if $t \in \operatorname{Max} \operatorname{Ind}(\mathcal{R}(\mathbf{x}))=\operatorname{Max} \operatorname{Ind}(\mathbf{x})$ then for all $k \in \mathbf{I}_{m}$

$$
\begin{aligned}
(\mathcal{R}(\mathbf{x}))_{t}-(\mathcal{R}(\mathbf{x}))_{k} & =\left(x_{t}-x_{k}\right)+ \\
& +\varepsilon\left(x_{t}-x_{k}\right)\left(\frac{x_{k}\left(x_{t}^{r} e^{\theta\left(1-x_{t}\right)}-x_{k}^{r} e^{\theta\left(1-x_{k}\right)}\right)}{x_{t}-x_{k}}+\sum_{i=1}^{m} x_{i}\left(x_{t}^{r} e^{\theta\left(1-x_{t}\right)}-x_{i}^{r} e^{\theta\left(1-x_{i}\right)}\right)\right) g(\mathbf{x})
\end{aligned}
$$

Since $\varepsilon<0$, we obtain that $\mathcal{M}_{k}(\mathcal{R}(\mathbf{x})) \leq \mathcal{M}_{k}(\mathbf{x})$ for all $k \in \mathbf{I}_{m}$.

By repeating this process, we get for all $k \in \mathbf{I}_{m}, n \in \mathbb{N}$, and $\mathbf{x} \in \operatorname{int} \mathbb{S}^{m-1}$ that

$$
\operatorname{Max} \operatorname{Ind}\left(\mathcal{R}^{(n)}(\mathbf{x})\right)=\operatorname{Max} \operatorname{Ind}(\mathbf{x}), \quad \mathcal{M}_{k}\left(\mathcal{R}^{(n+1)}(\mathbf{x})\right) \leq \mathcal{M}_{k}\left(\mathcal{R}^{(n)}(\mathbf{x})\right) .
$$

This shows that $\mathcal{M}_{k}(\mathbf{x})$ for $k \in \mathbf{I}_{m}$ is the Lyapunov function over the set int $\mathbb{S}^{m-1}$.

The part (ii) can be similarly proved. Its proof is omitted here.

We now prove the part (iii). Without loss of generality, we may assume that $\mathbf{x} \in$ int $\mathbb{S}^{m-1}$. Otherwise, we choose the suitable Lyapunov function given in the part ( $i i)$ depending on an initial point $\mathbf{x} \in \mathbb{S}^{m-1}$.

Since $\mathcal{M}_{k}(\mathbf{x})$ for $k \in \mathbf{I}_{m}$ is the Lyapunov function over the set int $\mathbb{S}^{m-1}$, one can show by using the same technique implemented in the part (iii) of Theorem 4.2 that the trajectory $\left\{\mathcal{R}^{(n)}(\mathbf{x})\right\}_{n=0}^{\infty}$ of the operator $\mathcal{R}: \operatorname{int} \mathbb{S}^{m-1} \rightarrow \operatorname{int} \mathbb{S}^{m-1}$ starting from any initial point $\mathbf{x} \in \operatorname{int} \mathbb{S}^{m-1}$ is convergent and its omega limiting point is some fixed (rest) point $\mathbf{x}^{* *}$. Moreover, we now show that the trajectory $\left\{\mathcal{R}^{(n)}(\mathbf{x})\right\}_{n=0}^{\infty}$ of the operator $\mathcal{R}: \operatorname{int} \mathbb{S}^{m-1} \rightarrow \operatorname{int} \mathbb{S}^{m-1}$ is separated from the boundary $\partial \mathbb{S}^{m-1}$ of the simplex $\mathbb{S}^{m-1}$, i.e., we have for some $\delta_{0}>0$ that

$$
\min _{k \in \mathbf{I}_{m}}\left(\mathcal{R}^{(n)}(\mathbf{x})\right)_{k} \geq \delta_{0}, \quad \forall n \in \mathbb{N}
$$

Indeed, as we already showed in the part $(i)$ that

$$
\operatorname{Sign}\left((\mathcal{R}(\mathbf{x}))_{t}-(\mathcal{R}(\mathbf{x}))_{k}\right)=\operatorname{Sign}\left(x_{t}-x_{k}\right)
$$

for any $k, t \in \mathbf{I}_{m}$, we obtain $\operatorname{Min} \operatorname{lnd}(\mathcal{R}(\mathbf{x}))=\operatorname{Min} \operatorname{lnd}(\mathbf{x})$ for all $\mathbf{x} \in \operatorname{int} \mathbb{S}^{m-1}$.

Since $\varepsilon<0$, it follows from (3.2) and $k \in \operatorname{MinInd}(\mathbf{x})$ that

$$
(\mathcal{R}(\mathbf{x}))_{k}=x_{k}\left[1-\varepsilon \sum_{i=1}^{m} x_{i}\left(x_{i}^{r} e^{\theta\left(1-x_{i}\right)}-x_{k}^{r} e^{\theta\left(1-x_{k}\right)}\right) g(\mathbf{x})\right] \geq x_{k} .
$$

This means that $\min _{k \in \mathbf{I}_{m}}(\mathcal{R}(\mathbf{x}))_{k} \geq \delta_{0}$ for $\delta_{0}:=\min _{k \in \mathbf{I}_{m}} x_{k}>0\left(\right.$ since $\left.\mathbf{x} \in \operatorname{int} \mathbb{S}^{m-1}\right)$.

By repeating this process, we obtain that

$$
\operatorname{Minlnd}\left(\mathcal{R}^{(n)}(\mathbf{x})\right)=\operatorname{Minlnd}(\mathbf{x}), \quad \min _{k \in \mathbf{I}_{m}}\left(\mathcal{R}^{(n)}(\mathbf{x})\right)_{k} \geq \delta_{0}, \quad \forall n \in \mathbb{N} .
$$


Since the trajectory $\left\{\mathcal{R}^{(n)}(\mathbf{x})\right\}_{n=0}^{\infty}$ of the operator $\mathcal{R}: \operatorname{int} \mathbb{S}^{m-1} \rightarrow \operatorname{int} \mathbb{S}^{m-1}$ is separated from the boundary $\partial \mathbb{S}^{m-1}$ of the simplex $\mathbb{S}^{m-1}$, its limiting point $\mathbf{x}^{* *}$ belongs to the interior int $\mathbb{S}^{m-1}$ of the simplex $\mathbb{S}^{m-1}$. Then the only fixed point in the interior int $\mathbb{S}^{m-1}$ of the simplex $\mathbb{S}^{m-1}$ is its center. Hence, the omega limiting point of the trajectory starting from any initial point $\mathbf{x} \in$ int $\mathbb{S}^{m-1}$ is the center of the simplex $\mathbb{S}^{m-1}$. This completes the proof.

\section{Conclusion}

The basis for evolutionary game theory is the Folk Theorem of Evolutionary Game Theory (see [5, 12]) which asserts the following three statements for all "reasonable" dynamics (particularly, the replicator equation) of an evolutionary game:

(i) A stable fixed (rest) point is a Nash equilibrium;

(ii) Any convergent trajectory evolves to a Nash equilibrium;

(iii) A strict Nash equilibrium is asymptotically stable.

In this paper, it has been shown that the Folk Theorem of Evolutionary Game Theory is also true for the model of evolutionary game in which the nonlinear payoff functions are defined by the Ricker models. In its own turns, this will immediately open up the door to study the dynamics and stability analysis of the replicator equation governed by the models of evolutionary game in which the nonlinear payoff functions are defined by other discrete population models for a single species.

\section{Acknowledgment}

A part of this work was presented at the Third International Conference on Mathematics and Statistics (AUS-ICMS'20), February 6-9, 2020, Sharjah, UAE and it was financially supported by American University of the Middle East, Kuwait. The author is greatly indebted to anonymous referee for carefully reading the manuscript and for providing such constructive comments and suggestions which substantially contributed to improving the quality and presentation of the paper.

\section{References}

[1] D. Acemoglu, A. Ozdaglar, Competition and efficiency in congested markets, Mathematics of Operations Research, 32(1), 1-31 (2007) (cited on page, 143, 144)

[2] D. Balkenborg, K. Schlag, Evolutionarily stable sets, Game Theory 29, 571-595 (2001) (cited on page, 142)

[3] M. Beckmann, C. B. McGuire, C. B. Winsten, Studies in the Economics of Transportation (Yale University Press, New Haven, 1956) (cited on page, 143, 144)

[4] R. Cressman, The stability concept of evolutionary game theory: A Dynamic Approach (SpringerVerlag, Berlin, Heidelberg, 1992) (cited on page, 139) 
[5] R. Cressman, Evolutionary dynamics and extensive form games. MIT Press, Cambridge (2003) (cited on page, 139, 153)

[6] S. Dafermos, F. Sparrow, The traffic assignment problem for a general network, Journal of Research of the National Bureau of Standards-B. Mathematical Sciences, 73(2), 91-118 (1969) (cited on page, 143, 144)

[7] D. Friedman, Evolutionary games in economics, Econometrica, 59(3), 637-666 (1991) (cited on page, 139)

[8] D. Friedman, On economic applications of evolutionary game theory, Journal of Evolutionary Economics, 8(1), 15-43 (1998) (cited on page, 139)

[9] R. Ganikhodzhaev, M. Saburov, A generalized model of the nonlinear operators of Volterra type and Lyapunov functions, J. Sib. Fed. Univ. Math. Phys. 1(2), 188-196 (2008) (cited on page, 146)

[10] J. Hofbauer, W. H. Sandholm, Stable games and their dynamics, Journal of Economic Theory, 144(4), 1665-1693 (2009) (cited on page, 146)

[11] J. Hofbauer, K. Sigmund, The theory of evolution and dynamical systems (Cambridge University Press, 1988) (cited on page, 139)

[12] J. Hofbauer, K. Sigmund, Evolutionary Games and Population Dynamics (Cambridge University Press, 1998) (cited on page, 139, 153)

[13] J. Hofbauer, K. Sigmund, Evolutionary game dynamics, Bull. Amer. Math. Soc. 40(4), 479-519 (2003) (cited on page, 139)

[14] T. Larsson, M. Patriksson, Price-directive traffic management: Applications of side constrained traffic equilibrium models, editors M. G. H. Bell, Transportation Networks: Recent Methodological Advances (Pergamon Press, Amsterdam, 1998), 83-97 (cited on page, 143, 144)

[15] T. Larsson, M. Patriksson, Side constrained traffic equilibrium models: Traffic management through link tolls, editors P. Marcotte, et al. Equilibrium and Advanced Transportation Model (Kluwer Academic Publishers, Boston, MA, 1998) 125-151 (cited on page, 143, 144)

[16] J. Maynard Smith, The theory of games and the evolution of animal conflicts, Journal of Theoretical Biology 47(1), 209-221 (1974) (cited on page, 139)

[17] J. Maynard Smith, Evolution and the Theory of Games. (Cambridge University Press, Cambridge, 1982) (cited on page, 139)

[18] J. Maynard Smith, G. R. Price, The logic of animal conflict, Nature, 246(5427), 15-18 (1973) (cited on page, 139)

[19] D. S. Mitrinovic, J. E. Pecaric, A. M. Fink, Classical and New Inequalities in Analysis. (Mathematics and Its Applications, Vol.61, Dordrecht: Kluwer Academic Publishers) (1993) (cited on page, 149, 151)

[20] J. F. Nash, Equilibrium points in n-person games, Proc. Nat. Acad. Sci. USA 36(1), 48-49 (1950) (cited on page, 139) 
[21] J. F. Nash, Non-cooperative games, Ann. Math. 54, 287-295 (1951) (cited on page, 139)

[22] M. A. Nowak, Evolutionary Dynamics: Exploring the Equations of Life (Harvard University Press, 2006) (cited on page, 139)

[23] A. Ozdaglar, R. Srikant, Incentives and pricing in communication networks, editors N. Nisan et al. Algorithmic Game Theory (Cambridge University Press, 2007) 571-591. (cited on page, 143, 144)

[24] M. Patriksson, The Traffic Assignment Problem: Models and Methods. (VSP, Utrecht, The Netherlands, 1994) (cited on page, 143, 144)

[25] H.-J. Pohley, B. Thomas, Non-linear ESS models and frequency dependent selection, BioSystems 16, 87-100 (1983) (cited on page, 141, 142)

[26] W. E. Ricker, Stock and recruitment, J. Fish. Res. Bd. Canada, 11, 559-623 (1954) (cited on page, 145)

[27] M. Saburov, On discrete-time replicator equations with nonlinear payoff functions, Dyn Games Appl (Submitted). (cited on page, 145)

[28] W. H. Sandholm, Potential games with continuous player sets, J Econ Theory 97, 81-108 (2001) (cited on page, 143)

[29] W. H. Sandholm, Evolutionary implementation and congestion pricing, Rev Econ Stud 69, 667-689 (2002) (cited on page, 143)

[30] W. H. Sandholm, Negative externalities and evolutionary implementation. Rev Econ Stud 72, 885915 (2005) (cited on page, 143)

[31] W. H. Sandholm, Population Games and Evolutionary Dynamics (MIT Press, Cambridge, 2010) (cited on page, 139, 143, 146)

[32] P. Schuster, K. Sigmund, Replicator dynamics, J Theor Biol 100(3), 533-538 (1983) (cited on page, 140)

[33] K. Sigmund, Evolutionary Game Dynamics: American Mathematical Society Short Course (Providence, Rhode Island, 2010) (cited on page, 139)

[34] J. Swinkels, Evolutionary stability with equilibrium entrants, Journal of Economic Theory, 57, 306-332 (1992) (cited on page, 142)

[35] P. D. Taylor, Evolutionarily stable strategies with two types of players, J. Appl. Probab. 16, 76-83 (1979) (cited on page, 140, 142)

[36] P. D. Taylor, L. Jonker, Evolutionarily stable strategies and game dynamics, Math. Biosci. 40, 145-156 (1978) (cited on page, 140, 142)

[37] B. Thomas, Evolutionary stability: states and strategies, Theor. Popul. Biol. 26, 49-67 (1984) (cited on page, 142) 
[38] B. Thomas, On evolutionarily stable sets, J. Math. Biol. 22, 105-115 (1985) (cited on page, 142)

[39] J. von Neumann, O. Morgenstern, Theory of games and economic behavior (Princeton University Press, 1944) (cited on page, 139)

[40] J. W. Weibull, Evolutionary Game Theory (MIT Press, Cambridge, 1995) (cited on page, 139) 